International Mathematical Forum, 2, 2007, no. 32, 1587 - 1603

\title{
Circulant Fractional Spline Wavelet Preconditioners for Solving Stochastic Heat Conduction Problem for Solving Weakly Singular Integral Equations
}

\author{
D. Rostamy V. F. \\ Department of Mathematics and Computer Science \\ Faculty of Science, University of International Imam Khomeini \\ \& University of Tehran, P.O. Box 14155-6455, Tehran, Iran \\ rostamy@khayam.ut.ac.ir, rostamyd@hotmail.com
}

\begin{abstract}
In this paper we consider a stochastic linear heat conduction problem that was reduced to a special weakly singular integral equation of the second kind. By using a practical variant of Galerkin boundary element method, circulant integral operators and the fractional spline wavelet bases, a new approach for solving of this problem is given. Numerical examples show that this approach is fast.
\end{abstract}

Mathematics Subject Classification: 65R20, 65Y10, 62L10, 62N99

Keywords: Stochastic integral equations, singular integral equation, numerical analysis

\section{INTRODUCTION}

In this paper two classes of preconditioners are proposed for solving stochastic integral equations. The first and second class of preconditioners are based on circulant operators. These equations arise in many of practical applications such as obstacle problems, heat conduction and dam type with noise. Since, we use the Galerkin method to approximate the solutions of these equations based on fractional spline wavelet bases. These bases can have effective properties. Let us consider the following equation:

$$
w(x)=T[\sigma(x)],
$$

such that $T[]=[]-\int_{G^{\prime}} a(x, y) k(x-y)[] d y$. Here $a \in C^{m}\left(G^{\prime} \times G^{\prime}\right)$, $k \in C^{m-1}\left(G^{\prime} \backslash\{0\}\right), m \geq 1, G^{\prime} \subseteq \mathbb{R}^{d}$ and $w$ a given process defined on a 
probability space (or a given function from a Banach space). This equation can be generated from a stochastic linear heat conduction such as:

$$
\left\{\begin{array}{l}
\nabla^{2} u-K^{2} u=\dot{W}(x, t) \\
\left.\frac{\partial u}{\partial n}\right|_{S}=0, \quad x \in G_{e} \subseteq \mathbb{R}^{d},
\end{array}\right.
$$

where $\dot{W}$ is a space-time noise and $S$ is the boundary of $G_{e}$ (exterior region to $S$ ) and $G_{i}$ is the interior region, while $n$ stands for the unit normally outwards to $S$, assuming $S$ is a smooth or regular boundary and $K$ is a constant [2, 3, $11,13,17,22,23,24,28]$. Suppose that $\mu$ be a $\sigma$-finite measure on $\mathbb{R}^{d-1}, \bar{\mu}$ the measure $d \mu \times d t$ on $\mathbb{R}^{d}$, and $W$ a Gaussian additive set function of the Borel sets of $\mathbb{R}^{d}$ such that $W(A)$ is a Gaussian random variable of mean zero and variance $\bar{\mu}(A)$ so that $W(A)$ and $W(B)$ are independent if $A \cap B=\phi$. In this case, the $x=\left(x_{1}, x_{2}, \ldots, x_{d-1}\right) \in \mathbb{R}^{d-1}$ and $W_{x t}=W\left\{\left(x_{1}, \infty\right) \times \ldots \times\left(x_{d-1},+\infty\right) \times(0, t)\right\}$ where $W$ is not differentiable, but its derivative can exist. If we consider $\dot{W}$ as $\frac{\partial^{d} W_{x, t}}{\partial x_{1} \ldots \partial x_{d-1} \partial t}$, then system (2) is very interesting when $d=3$, since the problem embodies the solution of an infinite expanse of material containing a cavity $S$ on which the temperature gradient is zero.

It is obvious the heat conduction problem is encapsulated by an integral equation if we assume that $u$ can be represented as the sum of a volume potential and a single-layer potential as follows:

$$
4 \pi u(x)=\int_{G_{e}} \dot{W}(\xi) E(x ; \xi) d V+\int_{S} \sigma(\xi) E(x ; \xi) d S,
$$

where $E(x, \xi)=\frac{\exp (-K|x-\xi|)}{|x-\xi|}$ is a fundamental solution of the Helmholtz equation, $\sigma$ is an unknown source density, with $x^{\prime}, \xi^{\prime} \in \mathbb{R}^{d-1}, x=\left(x^{\prime}, t\right), \xi=\left(\xi^{\prime}, t\right)$ and $|x|=\left(x_{1}^{2}+\ldots+x_{d-1}^{2}+t^{2}\right)^{\frac{1}{2}}$.

The next step is to take the normal derivative of both sides of equation (3), let $x$ approach $S$ and use the equations given in [20,21]. Then the result is a weakly singular integral equation of the second kind in $\sigma(x)$ for $(1)$ when $w(x)=\int_{G_{e}} \dot{W}(\xi) \frac{\partial E(x, \xi)}{\partial n} d V$ and $\int_{G^{\prime}} a(x, y) k(x-y) \sigma(y) d y$ is made from $\int_{S} \sigma(\xi) \frac{\partial E(x, \xi)}{\partial n} d S$ so that $G^{\prime}=\left\{x \in \mathbb{R}^{d}, 0 \leq x_{j}<\infty, j=1,2, \ldots, d\right\}$. (Of course, $w$ can be supposed to be a Wiener process defined on an appropriate probability space.)

In $[20,21]$, we have used the collocation method based on b-spline bases but here the Galerkin method based on fractional spline wavelet are used.

The outline of the paper is as follows. In section 2 Galerkin boundary element methods is discussed by fractional spline wavelet bases. In section 3, we 
rewrite the integral equation by circulant operators as preconditioners similar to those that will be constructed afterwards. The convergence analysis of the preconditioned operators are discussed in section 3 and we give an important theorem for stability. Finally, in section 4, numerical methods involving preconditioned conjugated gradient method and employing Gaussian quadrature rules as numerical algorithms are also investigated.

\section{GALERKIN BOUNDARY ELEMENT METHODS BY FRACTIONAL SPLINE WAVELET BASES}

Consider the Fractional Spline Wavelet (FSW) bases

$$
\left[\begin{array}{l}
\psi_{+}^{\alpha+i}(x / 2)=\sum_{k \in Z} \frac{(-1)^{k}}{2^{\alpha+i}} \sum_{l \in Z}\left(\begin{array}{c}
\alpha+i+1 \\
l
\end{array}\right) \beta_{*}^{2 \alpha+i+1}(l+k-1) \beta_{+}^{\alpha+i}(x-k), \\
i=0,1,2, \ldots
\end{array}\right.
$$

where $\beta^{\alpha}(x)$ is a fractional B-spline with degree $\alpha$. Also, we define the fractional causal B-splines by taking $(\alpha+1)$ th fractional difference of the one side power function

$$
\beta_{+}^{\alpha}(x)=\frac{\Delta_{+}^{\alpha+1} x_{+}^{\alpha}}{\Gamma(\alpha+1)}=\frac{1}{\Gamma(\alpha+1)} \sum_{k=0}^{+\infty}\left(\begin{array}{c}
\alpha+1 \\
k
\end{array}\right)(x-k)_{+}^{\alpha}
$$

and

$$
\beta_{*}^{\alpha}(x)=\frac{\Delta_{+}^{\alpha+1}|x|_{*}^{\alpha}}{\Gamma(\alpha+1)}=\frac{1}{\Gamma(\alpha+1)} \sum_{k \in Z}\left(\begin{array}{c}
\alpha+1 \\
k+\frac{\alpha+1}{2}
\end{array}\right)|x-k|_{*}^{\alpha},
$$

where $\alpha>\frac{-1}{2}$ in order to ensure square integrability. These functions interpolate the usual polynomial B-splines; these are recovered for $\alpha$ integer. They are "causal" in the sense that their support belong to $\mathbb{R}^{+}$. Here, we assume that the fractional has been extended to non-integer $\alpha$ by $\alpha !=\Gamma(\alpha+1)$ using Euler's gamma function.

Here $\Delta_{+}^{\alpha} f(x)=\sum_{k=0}^{+\infty}(-1)^{\alpha}\left(\begin{array}{c}\alpha+1 \\ k\end{array}\right) f(x-k)$,

$x_{+}^{\alpha}=\left\{\begin{array}{ll}x^{\alpha} & \alpha \geq 0 \\ 0 & \text { otherwise }\end{array}\right.$ and

$|x|_{*}^{\alpha}=\left\{\begin{array}{lll}\frac{|x|^{\alpha}}{-2 \sin \left(\frac{\pi}{2}\right)} & \text { if } \quad \alpha \text { not even } \\ \frac{x^{2 n} \log x}{(-1)^{1+n} \pi} & \text { if } \quad \alpha=2 n \text { (even) }\end{array}\right.$.

As $S_{N}^{\alpha}$ is subspace of $L^{2}(\Gamma)$, we can approximate the above boundary integral equations with Galerkin method. It is clear that $\psi_{+}^{\alpha+i}$ is a Riesz basis for $L^{2}(\Gamma)[26,27]$. As the degree $\alpha$ increases, the functions converge to modulated Gaussian which are known to optimally time-frequency localized in sense 
of Eisenberh uncertainty principle. This limit behavior can be inferred from the general convergence theorem in [1]. We use test and trial functions in these finite dimensional subspace and obtain the following Galerkin approximations. Unser and Blu (see [26, 27]) have shown that the presence of the B-spline convolution factor explains all key wavelet properties such as: order of approximation, reproduction of polynomials, vanishing moments, multi-scale differentiation property, and smoothness of the basis functions. Also, they have studied about the B-spline factorization and stability of wavelet bases with respect to differentiation.

First, we have the standard forms which starts with the decomposition of $\sigma$ and $w$ in a fractional wavelet basis form, by using $P_{n}$ as projection method:

$$
\begin{aligned}
& P_{n} \sigma=\sum_{j=0}<\sigma, \psi_{+}^{\alpha+j}>\psi_{+}^{\alpha+j}, \\
& P_{n} w=\sum_{j=0}<w, \psi_{+}^{\alpha+j}>\psi_{+}^{\alpha+j} .
\end{aligned}
$$

Hence, we have:

$$
T P_{n} \sigma=\sum_{j=0}<\sigma, \psi_{+}^{\alpha+j}>T \psi_{+}^{\alpha+j}
$$

Also, we have

$$
T \psi=\sum_{j=0}<T \psi, \psi_{+}^{\alpha+j}>\psi_{+}^{\alpha+j}
$$

which gives:

$T P_{n} \sigma=\sum_{j=0} \sum_{k=0}<T \psi_{+}^{\alpha+k}, \psi_{+}^{\alpha+j}><\sigma, \psi_{+}^{\alpha+k}>\psi_{+}^{\alpha+j}=\sum_{k=0}<w, \psi_{+}^{\alpha+k}>\psi_{+}^{\alpha+k}$,

where $<.$, . $>$ denotes an inner product.

Remark 1. In general, for $\Omega \subseteq \mathbb{R}^{d}$ such that $d \geq 2$, we can write $P_{n} \sigma=$ $\sum a_{j_{1} \ldots j_{n}} \Pi_{k=1}^{j_{n}} \psi_{+}^{\alpha_{k}}$ where $\otimes$ is the tensor product for producing the space of $V_{j_{1}} \otimes \ldots \otimes V_{j_{n}}$ and $a_{j_{1} \ldots j_{n}}$ are unknown coefficients $[4,16]$.

Therefore, (5) can be depicted by on infinity matrix whose coefficients are

$$
a_{k j}=<T_{i} \psi_{+}^{\alpha+k}, \psi_{+}^{\alpha+j}>\psi_{+}^{\alpha+j},
$$

and the following system is obtained:

$$
A_{n}^{\alpha} x=b
$$


Where

$$
A_{n}^{\alpha}=\left(a_{k j}=<T \psi_{+}^{\alpha+k}, \psi_{+}^{\alpha+j}>\psi_{+}^{\alpha+j}\right)_{k, j=0}^{n}
$$

and

$$
x=\left(<\sigma, \psi_{+}^{\alpha+k}>\right), \quad b=\left(<w, \psi_{+}^{\alpha+k}>\psi_{+}^{\alpha+k}\right) .
$$

Therefore, we observe that the equation (1) can have recourse to the finite systems that is (6) we call this transformation finite section method. Let $\left\{A_{n}^{\alpha}\right\}_{n=1}^{\infty}$ be a sequence on $n \times n$ matrices $A_{n}^{\alpha}$. This sequence is said to be stable if there is an $n_{0}$ such that the matrices $A_{n}^{\alpha}$ are invertible for all $n \geq n_{0}$ and

$$
\sup _{n \geq n_{0}}\left\|\left(A_{n}^{\alpha}\right)^{-1}\right\|<\infty .
$$

Using the convention to put $\|T\|=\infty$ if $T$ is not invertible, we can say that $\left\{A_{n}^{\alpha}\right\}_{n=1}^{\infty}$ is a stable sequence if and only if

$$
\lim \sup _{n \rightarrow \infty}\left\|\left(A_{n}^{\alpha}\right)^{-1}\right\|<\infty
$$

stability plays a central role in asymptotic linear algebra and numerical analysis. The our aim is to confine ourselves to part stability that is plays in connection with the finite section method.

One standard way for speeding up the convergence rate and stability is to apply a preconditioner so that it causes to cluster the spectrum around one. But, how can we make a continuous preconditioner such that the spectrum of matrix is clustered? For answering to this question the following section is provided.

\section{CIRCULANT WAVELET INTEGRAL OPERATORS}

Regarding the solution smoothness two ideas for the rate of convergence can be stated $[20,21]$ :

a) The use of circulant preconditioned operators that could increase the rate of convergence of the conjugate gradient method [8,25].

b) The use of special bases can ensure that the rate of convergence of the Galerkin method is optimal in terms of the uniform norm $[3,5,7,9,12,18]$. In this section, the approximate solution is studied by combining these two ideas, i.e. circulant integral operators and fractional spline wavelet bases for making of circulant wavelet operators.

3.1. Preliminary. One way of solving (1) is to use the projection method $[6,12]$, where the solution $\sigma(x)$ of $(1)$ is approximated by the solution $\sigma_{\tau}(x)$ of the equation

$$
\sigma_{\tau}(x)=\int_{G^{\prime \prime}} a(x, y) k(x-y) \sigma_{\tau}(y) d y+w(x)
$$


where $G^{\prime \prime}=\left\{x \in G^{\prime} \mid 0 \leq x_{j}<\tau, j=1,2, \ldots, d\right\}[6,7,8]$. It is clear that $\lim _{\tau \rightarrow \infty}\left\|\sigma_{\tau}-\sigma\right\|_{L_{p}\left(G^{\prime \prime}\right)}=0$ for $1 \leq p<\infty$. If we define

$$
A_{\tau}[]= \begin{cases}\int_{G^{\prime \prime}} a(x, y) k(x-y)[] d y, & x \in G^{\prime \prime} \backslash\{x=y\} \\ 0 & x \notin G^{\prime \prime} \backslash\{x=y\}\end{cases}
$$

then $A_{\tau}[]$ is a compact operator and $A_{\tau}: L_{p}\left(G^{\prime \prime} \backslash\{x=y\}\right) \rightarrow E_{\tau}^{\beta, m}$, here we assume:

$$
E_{\tau}\left(G^{\prime}\right)=E_{\tau}^{\beta, m}=\left\{\sigma \in C\left(G^{\prime}\right) \cap C^{m}\left(G^{\prime}\right) \mid \sum_{|\beta| \leq m} \sup _{x \in G^{\prime}} \frac{\left|D^{|\beta|} \sigma(x)\right|}{|x|^{-|\beta|}+(|\tau-| x||)^{-|\beta|}}<\infty\right\}
$$

equipped with the norm

$$
\|\sigma\|_{E_{\tau}\left(G^{\prime}\right)}=\max _{x \in G^{\prime}}|\sigma(x)|+\sum_{|\beta| \leq m} \sup _{x \in G^{\prime}} \frac{\left|D^{|\beta|} \sigma(x)\right|}{|x|^{-|\beta|}+(|\tau-| x||)^{-|\beta|}}
$$

is a Banach space (see [20]) where $0<\tau \leq \infty$ and $x=\left(x_{1}, \ldots, x_{d}\right) \in G^{\prime}$.

The spectrum of the operator $T_{\tau}=I-A_{\tau}$ is clustered around 1 because that is a compact operator (where $I$ is the identity operator). Therefore solving (9) by iterative methods such as the Conjugate Gradient (CG) method will be less expensive than using direct methods $[12,15,21]$. One standard way for speeding up the convergence rate of the $\mathrm{CG}$ method is to apply a preconditioner. As $\tau \rightarrow \infty$, the spectrum of the operator $A_{\tau}$ becomes dense in the spectrum of the operator defined by (1). Hence the convergence rate of the $\mathrm{CG}$ will deteriorate [8].

Remark 2. It is clear that the iterations $\sigma_{k}, k=1,2, \ldots$ of the $\mathrm{CG}$, in general, converge to the solution $\sigma_{\tau}$ of (9) with a linear rate of convergence $\left\|\sigma_{\tau}-\sigma_{k}\right\|_{E_{\tau}\left(G^{\prime}\right)} \leq c\left(\frac{l-1}{l+1}\right)^{k}$. Where $c$ is positive constant and the $\frac{l-1}{l+1}$ depends on the condition number of the operator $A_{\tau}$ (i.e. $l=l\left(A_{\tau}\right)=$ $\left.\sqrt{\left\|A_{\tau}\right\|\left\|A_{\tau}^{-1}\right\|}\right)[8,15]$.

Thus instead of solving (9), we propose two ways based on the preconditioner idea. If we put $S T_{\tau}=\left(I+C_{\tau}\right)^{-1}\left(I-A_{\tau}\right)$ and $w_{\tau}=\left(I+C_{\tau}\right)^{-1} w$ then the first way is as follows:

$$
S T_{\tau} P_{n} \sigma=\sum_{j=0} \sum_{k=0}<S T_{\tau} \psi_{+}^{\alpha+k}, \psi_{+}^{\alpha+j}><\sigma, \psi_{+}^{\alpha+k}>\psi_{+}^{\alpha+j}=\sum_{k=0}<w_{\tau}, \psi_{+}^{\alpha+k}>\psi_{+}^{\alpha+k}
$$


where the operator $C_{\tau}$ is a preconditioner for the operator $A_{\tau}$. Also, if we put $O T_{\tau}=\left(I+C_{\tau}\right)$ then the second way is as follows:

$O T_{\tau} P_{n} \sigma=\sum_{j=0} \sum_{k=0}<O T_{\tau} \psi_{+}^{\alpha+k}, \psi_{+}^{\alpha+j}><\sigma, \psi_{+}^{\alpha+k}>\psi_{+}^{\alpha+j}=\sum_{k=0}<w, \psi_{+}^{\alpha+k}>\psi_{+}^{\alpha+k}$

where the circulant integral operator is a good preconditioner of $C_{\tau}[]$ in the sense that it is close to $A_{\tau}[]$ in some norm.

Remark 3. We remark that the following bound for the convergence of the PCG algorithm that may be applied to solve $C^{-1} A_{n}^{\alpha} x=C^{-1} b$ :

$$
\left\|\sigma_{\tau}-\sigma_{k}\right\|_{E_{\tau}\left(G^{\prime}\right)} \leq c_{1}\left(\frac{\epsilon}{1+\sqrt{1-\epsilon^{2}}}\right)^{k}, \quad c_{1}=\text { constant }
$$

if the spectral elements of $C^{\frac{-1}{2}} A_{n}^{\alpha} C^{\frac{-1}{2}}$ are contained in $(1-\epsilon, 1+\epsilon)$ with $\epsilon<1$ (see $[8,15])$.

Usually the equation (11) is rather easier to solve than equation (10). Let us introduce $\mathcal{G}=\left\{C_{\tau} \mid C_{\tau}[]=\int_{G^{\prime \prime}} h_{\tau}(x-y)[] d y, \quad x, y \in G^{\prime \prime} \backslash\{x=y\}\right\}$ as a class of preconditioners (where $h_{\tau}$ is the periodic function in $G^{\prime \prime} \backslash\{x=y\}$ and conditions $(4),(5)$ in $[20,21]$ are satisfied for it). Choosing $C_{\tau}[]$ is based on two standard way:

(i) to minimize $\left\|A_{\tau}-C_{\tau}\right\|^{2}$

(ii) to minimize $\left\|I-\left(I+C_{\tau}\right)^{-1}\left(I-A_{\tau}\right)\right\|^{2}$

on $\mathcal{G}$ with the Hilbert-Schmidt norm, where the first circulant integral is called the optimal circulant integral and the second is the called the super-optimal circulant integral. In [8] the authors assumed that in (9), $a(x, y)=1$ and the kernel does not have a singularity but here it is supposed that $a(x, y) \neq 1$ and the kernel function has a singularity.

\subsection{Construction of Optimal Circulant Integral By Wavelet Se- ries.}

If the first minimizer is called the optimal preconditioner and is denoted by $p\left(A_{\tau}\right)$, then it is possible to construct the optimal circulant integral preconditioner $p\left(A_{\tau}\right)$ for integral operators $A_{\tau}$. The preconditioner $p\left(A_{\tau}\right)$ is defined to be the circulant integral operator that minimizes the Hilbert-Schmidt norm

$$
\left\|A_{\tau}-H_{\tau}\right\|^{2}=\int_{G^{\prime \prime}} \int_{G^{\prime \prime}}\left(a(x, y) k(x-y)-h_{\tau}(x-y)\right)\left(\bar{a}(x, y) \bar{k}(x-y)-\bar{h}_{\tau}(x-y)\right) d y d x
$$

over all circulant integral operators $C_{\tau}$ or $\mathcal{G}$.

The expression of the kernel function of $p\left(A_{\tau}\right)$ is presented first. Since 
$a, k \in L_{p}\left(G^{\prime \prime} \backslash\{x=y\}\right)$, by using the wavelet series we have

$$
a(x, y) k(x-y)=\sum_{m, n=-\infty}^{\infty} v_{m, n} \psi_{+}^{\alpha+m}(x) \psi_{+}^{\alpha+n}(y), \quad x \neq y
$$

where $\psi_{+}^{\alpha+m}$ is an eigenfunction of the operator $C_{\tau}$ with the following eigenvalue:

$$
\lambda_{m}=<h_{\tau}, \psi_{+}^{\alpha+m}>=\int_{G^{\prime \prime}} h_{\tau}(x) \psi_{+}^{\alpha+m}(x) d x, \quad m \in Z,
$$

and

$$
\begin{gathered}
v_{m, n}=\int_{G^{\prime \prime}} \int_{G^{\prime \prime}} a(x, y) k(x-y) \psi_{+}^{\alpha+n}(y) \psi_{+}^{\alpha+m}(x) d x d y \\
=<A_{\tau} \psi_{+}^{\alpha+n}, \psi_{+}^{\alpha+m}>, \quad m, n \in Z .
\end{gathered}
$$

By means of the wavelet series, we can write

$$
h_{\tau}(x-y)=\sum_{m=-\infty}^{\infty} \lambda_{m} \psi_{+}^{\alpha+m}(x) \psi_{+}^{\alpha+m}(y), \quad x, y \in G^{\prime \prime} \backslash\{x=y\} .
$$

Combining this equation with (13) and using the orthonormal bases of $\psi_{+}^{\alpha+n}$ (see $[26,27]$ ), we can express the distance (15) as

$$
\left\|A_{\tau}-C_{\tau}\right\|^{2}=\sum_{m=-\infty}^{+\infty}\left|v_{m, m}-\lambda_{m}\right|^{2}+\sum_{\substack{m, n=-\infty \\ m \neq n}}^{\infty}\left|v_{m, n}\right|^{2} .
$$

Clearly, the expression becomes minimal if and only if $\lambda_{m}=v_{m, m}=<$ $A_{\tau} \psi_{+}^{\alpha+m}, \psi_{+}^{\alpha+m}>$ for all integers $m$.

\subsection{Construction of Super-Optimal Circulant Integral By Wavelet Series.}

This section is an extension of an idea presented by Tyrtyshikov and Strela [25]. We consider another type of circulant integral operator which is obtained by minimizing the Hilbert-Schmidt norm

$$
\left\|I-\left(I+C_{\tau}\right)^{-1}\left(I-A_{\tau}\right)\right\|^{2}
$$

over all circulant integral operators of $\mathcal{G}$ such that the operator $\left(I+C_{\tau}\right)^{-1}$ exists. Let $C_{\tau}[]$ be a circulant integral operator with kernel function and eigenvalues $\lambda_{m}$ given in (14). If $\left(I+C_{\tau}\right)$ [ ] is invertible, then $1+\lambda_{n} \neq 0$ for all integers $n$. Moreover, since $h_{\tau}$ is in $L_{2}\left(G^{\prime \prime} \backslash\{x=y\}\right)$ and $\sum_{n=-\infty}^{\infty}\left(\lambda_{n}\right)^{2}<\infty$ , therefore $\left|1+\lambda_{n}\right| \geq 1 / 2$ for all $|n|$ that are sufficiently large. On the other 
hand $\left(I+C_{\tau}\right)^{-1}=I-K_{\tau}$, where $K_{\tau}$ is also a circulant integral operator with kernel function

$$
k_{\tau}(x-y)=\sum_{n=-\infty}^{+\infty} \frac{-\lambda_{n}}{1+\lambda_{n}} \psi_{+}^{\alpha+n}(x) \psi_{+}^{\alpha+n}(y), \quad x \neq y .
$$

Since, the kernel function of $C_{\tau} K_{\tau}$ at the point $(t-s)$ is given by

$$
\begin{gathered}
\int_{G^{\prime \prime}} \sum_{m=-\infty}^{+\infty} \lambda_{m} \psi_{+}^{\alpha+m}(t) \psi_{+}^{\alpha+m}\left(y^{\prime}\right) \sum_{n=-\infty}^{+\infty} \frac{-\lambda_{n}}{1+\lambda_{n}} \psi_{+}^{\alpha+n}\left(y^{\prime}\right) \psi_{+}^{\alpha+n}(s) d y^{\prime} \\
=\sum_{n=-\infty}^{\infty} \frac{-\lambda_{n}^{2}}{1+\lambda_{n}} \psi_{+}^{\alpha+n}(t) \psi_{+}^{\alpha+n}(s)
\end{gathered}
$$

the function $k_{\tau}$ is a $\tau$-periodic function and it is straightforward to check the kernel function $C_{\tau}-K_{\tau}-C_{\tau} K_{\tau}=\left(I+C_{\tau}\right)\left(I-K_{\tau}\right)-I$.

The problem of minimizing the norm (16) becomes the problem of minimizing $\left\|I-\left(I-K_{\tau}\right)\left(I-A_{\tau}\right)\right\|$ over all circulant integral operators $K_{\tau}$. We assume the $v_{n, m}=<A_{\tau} \psi_{+}^{\alpha+n}, \psi_{+}^{\alpha+m}>$ and $\xi_{m}=\sum_{n=-\infty}^{+\infty}\left|v_{n, m}\right|^{2}$. Then, $\left\|I-\left(I-K_{\tau}\right)\left(I-A_{\tau}\right)\right\|=\left\|K_{\tau}+A_{\tau}-K_{\tau} A_{\tau}\right\|$ and, by using (17), the kernel function of $K_{\tau}-K_{\tau} A_{\tau}+A_{\tau}$ at point $(x, y)$ is given by

$$
\begin{gathered}
\sum_{m, n=-\infty}^{\infty}\left\{-\frac{\delta_{m, n} \lambda_{m}}{1+\lambda_{m}}-\frac{\lambda_{m} v_{m, n}}{1+\lambda_{m}}+v_{m, n}\right\} \psi_{+}^{\alpha+m}(x) \psi_{+}^{\alpha+n}(y)= \\
=\sum_{m, n=-\infty}^{\infty}\left(\frac{-\delta_{m, n} \lambda_{m}+v_{m, n}}{1+\lambda_{m}}\right) \psi_{+}^{\alpha+m}(x) \psi_{+}^{\alpha+n}(y),
\end{gathered}
$$

where $\delta_{m, n}$ denotes the Kronecker symbol. By the definition of the HilbertSchmidt norm,

$$
\left\|I-\left(I+C_{\tau}\right)^{-1}\left(I-A_{\tau}\right)\right\|^{2}=\sum_{m, n=-\infty}^{+\infty}\left|\frac{\delta_{m, n} \lambda_{m}-v_{m, n}}{1+\lambda_{m}}\right|^{2},
$$

it is clear that the above expression is minimized if and only if the term $\frac{\left|\lambda_{m}\right|^{2}-\lambda_{m} \bar{v}_{m, m}-\lambda_{m} v_{m, m}+\xi_{m}}{\left|1+\lambda_{m}\right|^{2}}$ is minimized for all integers $m$. However, by differentiating this quotient with respect to the real and imaginary parts of $\lambda_{m}$, we see that minimum will be obtained if we set $\lambda_{m}=\frac{\xi_{m}+v_{m, m}}{1+v_{m, m}}$ for all $m$. Hence, the kernel function of super-optimal circulant $C_{\tau}[]$ is given by:

$$
c_{\tau}(x-y)=\sum_{m=-\infty}^{+\infty}\left(\frac{\xi_{m}+v_{m, m}}{1+v_{m, m}}\right) \psi_{+}^{\alpha+m}(x) \psi_{+}^{\alpha+m}(y), \quad x \neq y .
$$


Moreover, since $1+\lambda_{m}=\frac{\sum_{n=-\infty}^{+\infty}\left|v_{m, n}\right|^{2}+\left|1+v_{m, m}\right|^{2}}{1+v_{m, m}} \neq 0$, we can see that $\left(I+C_{\tau}\right)[]$ is invertible.

Remark 4. Let $\mathbf{A}, \mathbf{C}$ be as the matrices that has been discritized from the operators of (9) and (11) respectively. Then $\mathbf{C}$ for $\mathbf{A}$ can be viewed as an approximation to $\mathbf{A}$ and can be used to obtain the following iterative method for solving the system (9) or $\mathbf{A x}=\mathbf{w}$. The convergence of this iteration (or an acceleration of it by conjugate gradient or other methods) depends on the spectrum of $\mathbf{C}^{-\mathbf{1}}(\mathbf{C}-\mathbf{A})$ : the smaller $\lambda\left(\mathbf{C}^{-\mathbf{1}}(\mathbf{C}-\mathbf{A})\right)$, the faster the convergence. It can be easily shown that, if $\mathbf{B}=\mathbf{C}-\mathbf{A}$, then

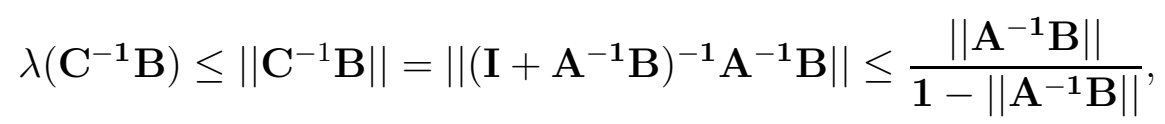

provided $\left\|\mathbf{A}^{-\mathbf{1}} \mathbf{B}\right\|<1$ in some consistent matrix norm. Since the upper bound is a strictly increasing function of $\left\|\mathbf{A}^{-\mathbf{1}} \mathbf{B}\right\|$, it is natural to chose $\mathbf{C}$ such that $\left\|\mathbf{A}^{-\mathbf{1}} \mathbf{B}\right\|$ is minimized. In general, the convergence rate of the conjugate gradient iteration depends on the spectrum and this problem is studied in detail in more recent papers (for example see $[9,15,25]$ ). Therefore, comparing the convergence rate of (11) with (9) is clear.

On the other hand, we can have a remark similar to this for (10), (9) in the end of section 3.3 that we dispense with it.

According to Remarks 4, 3 and equations (9), (10), we can say that the rates of convergence for the optimal and super-optimal preconditioners are fast. For proving our claim, we bring the following section.

\subsection{Convergence Analysis.}

In [20], we analyzed the convergence rate of the optimal and super-optimal circulant integrals as preconditioners for solving the integral equation of the second kind. We give a new theorem for stability and expand the theorems by Rostami et al. in the following.

Let us $S$ be an operator so that (1) is transferred to

$$
S T \sigma=S w
$$

Therefore, for stability, we can state the following theorem:

Theorem 1. Let $\left\{A_{n}^{\alpha}\right\}$ be a sequence of $n \times n$ matrices for $\alpha>\frac{-1}{2}$ and suppose there is an operator $S T$ such that $C_{n}^{-1} A_{n}^{\alpha} \rightarrow S T$ and $\left(C_{n}^{-1} A_{n}^{\alpha}\right)^{*} \rightarrow$ $(S T)^{*}$ strongly. If $\left\{C_{n}^{-1} A_{n}^{\alpha}\right\}$ is stable, then $S T$ is necessarily invertible and

$$
\left\|(S T)^{-1}\right\| \geq \liminf _{n \rightarrow \infty}\left\|\left(A_{n}^{\alpha}\right)^{-1} C_{n}\right\| .
$$


Proof. Suppose $\left\|\left(A_{n}^{\alpha}\right)^{-1} C_{n}\right\| \geq M$ for infinitely many $n$ and $P_{n}$ be the following projection:

$$
P_{n}: l^{2} \rightarrow l^{2}, \quad\left(x_{0}, x_{1}, x_{2}, \ldots\right) \rightarrow\left(x_{0}, x_{1}, x_{2}, \ldots, x_{n-1}, 0,0, \ldots\right) .
$$

For $x \in l^{2}$ and these $n$,

$$
\begin{gathered}
\left\|P_{n} x\right\|=\left\|\left(A_{n}^{\alpha}\right)^{-1} C_{n} C_{n}^{-1} A_{n}^{\alpha} P_{n} x\right\| \leq M\left\|C_{n}^{-1} A_{n}^{\alpha} P_{n} x\right\| \\
\left\|P_{n} x\right\|=\left\|\left(\left(C_{n}^{-1} A_{n}^{\alpha}\right)^{*}\right)^{-1}\left(C_{n}^{-1} A_{n}^{\alpha}\right)^{*} P_{n} x\right\| \leq M\left\|\left(C_{n}^{-1} A_{n}^{\alpha}\right)^{*} P_{n} x\right\|
\end{gathered}
$$

and passing to the limit $n \rightarrow \infty$, we get

$$
\|x\| \leq M\|S T x\|, \quad\|x\| \leq M\left\|(S T)^{*} x\right\|,
$$

which implies that $S T$ is invertible and $\left\|(S T)^{-1}\right\| \leq M$. Here $S$ can be as a continues Circulant Fractional Spline Wavelet(CFSW) operator .

Also, we recall and expand the following theorems that are hold for CFSW operators.

Theorem 2. Let $A$ be a self-adjoint operator with the kernel function a $(x, y) k(x-$ $y)=k_{1}(x-y)+a_{1}(x, y)$ where $k_{1}(.) \in C^{m-1}\left(G^{\prime} \backslash\{0\}\right) \cap L_{2}\left(G^{\prime}\right)$ and $a_{1}(x, y)$ satisfies $\int_{G^{\prime}} \int_{G^{\prime}}\left|a_{1}(x, y)\right|^{2} d x d y \leq M<\infty, \quad M=$ constant. Let $A_{\tau}$ be the defined operator in section 3.1 and $C_{\tau}$ be the optimal CFSW operator as preconditioner for $A_{\tau}$. Then for each $\epsilon>0$, there is $a \rho \in \mathbb{N}$ and $a \tau^{\star}>0$ with the following properties:

(i) for each $\tau \geq \tau^{\star}$ there exists a decomposition $A_{\tau}-C_{\tau}=R_{\tau}+E_{\tau}$ where $R_{\tau}$ and $E_{\tau}$ are self-adjoint operators.

(ii) The spectrum of $\left(I+C_{\tau}\right)^{\frac{-1}{2}}\left(I-A_{\tau}\right)\left(I+C_{\tau}\right)^{\frac{-1}{2}}$ has at most $\rho$ eigenvalues outside the interval $(1-\epsilon, 1+\epsilon)$ whenever $\tau \geq \tau^{\star}$.

Proof. See Rostami et al., (see [20]).

This theorem basically states that the spectrum of the CFSW operator as preconditioned operator is clustered around 1. Hence, if $I-A_{\tau}$ is preconditioned by $I+C_{\tau}$, we expect fast convergence. We now give the following theorem for the super-optimal preconditioner. Finally, Theorem 3 will hold for the super-optimal circulant as a preconditioner.

Theorem 3. Let all of the assumptions of theorem 1 satisfy. Moreover, we denote $O_{\tau}$ and $S_{\tau}$ as the optimal and super-optimal preconditioners (CFSW operators), respectively. Then we have:

(a) $\lim _{\tau \rightarrow \infty}\left\|O_{\tau}-S_{\tau}\right\|_{2}=0$, 
(b) Theorem 2 is valid for the super-optimal preconditioner.

Proof (a). It is clear that for each $\epsilon_{1}, \epsilon_{2}>0$ and by using the results of sections 3.2 and 3.3 , we have:

$$
0 \leq \lim _{\tau \rightarrow \infty}\left\|O_{\tau}-S_{\tau}\right\|_{2} \leq \lim _{\tau \rightarrow \infty}\left\|A_{\tau}-O_{\tau}\right\|_{2}+\lim _{\tau \rightarrow \infty}\left\|A_{\tau}-S_{\tau}\right\|_{2} \leq \epsilon_{1}+\epsilon_{2} \leq 2 \epsilon_{1}
$$

if $\epsilon_{1}=\epsilon_{2}$.

Proof (b). We can state; there is a $\rho \in \mathbb{N}$ and a $\tau^{\star}>0$ with the following properties:

(i) for each $\tau \geq \tau_{1}^{\star}$ there exists a decomposition $A_{\tau}-O_{\tau}=R_{\tau}+E_{\tau}$ where $R_{\tau}$ and $E_{\tau}$ are self-adjoint operators.

(ii) for each $\tau \geq \tau_{1}^{\star}$ there exists a decomposition $A_{\tau}-S_{\tau}=K_{\tau}+H_{\tau}$ where $K_{\tau}$ and $H_{\tau}$ are self-adjoint operators.

Proof (a) shows that (i) and (ii) are equivalent.

\section{NUMERICAL EXPERIMENTS}

We consider the algorithms called Alg.W, Alg.S and Alg.O. Here all of integrals are computed by the Gaussian quadrature rule. Consequently, every one of equations (6), (10) and (11) is replaced by a system of linear equations. For solving this linear system, the CG can be used. Hence, the algorithm Alg. W is the CG algorithm without a preconditioner, while algorithms Alg.O and Alg.S denote the circulant wavelet optimal preconditioner by PCG (letter O) and circulant wavelet Super-optimal preconditioner by PCG (the first letter $\mathrm{S})$ respectively.

Remark 5. In the examples, the zero vector is used as our initial guess and the stopping criterion is $\left\|r_{k}\right\| /\left\|r_{0}\right\|<10^{-7}$ where $r_{k}$ is the residual vector of the CG and PCG methods after $\mathrm{k}$ iterations.

Remark 6. On the right hand side of equations (6), (10) and (11) the function $w(x)=w_{n}$ may be a stochastic integral. So, it can be generated as a random function (that is a random number $\times$ error function) (see $[2,5,14,19$, $22,23,28])$. In the examples, the performance of linear systems with normally distributed random variables as white noise is first tested, in which $E\left(w_{n}\right)=0$ and $E\left(\left(w_{n}\right)^{2}\right)=\xi_{i}$ for each iteration.

At present, two numerical examples for testing the convergence performance and stability of the algorithms are given. The following examples are similar 
to [20] because we want to compare our results about this problem.

Example 1. In the singular integral equation of the second kind

$$
\sigma(x)=\int_{G} K(x, y) \sigma(y) d y+w(x)
$$

the kernel $K(x, y)=a(x, y) \log (|x-y|)$, where $x, y \in \mathbb{R}^{2}$, satisfies (5) with $v=0$ if $a(x, y)$ is $m$ times continuously differentiable on $(G \times G) \backslash\{x=y\}$. The numerical solutions are constructed by some approximate solution $\sigma_{\tau, n}$ to the integral equation (20). The dominant $G$ is a part of circle and its boundary $S$ is $\rho(\varphi)=(b \cos (\varphi), b \sin (\varphi))$ where $0 \leq b \leq \infty$ and $0 \leq \varphi \leq \frac{\pi}{4}$. Tables 1 , 2 show the numbers of iterations required for the convergence performance of the algorithms when $a(x, y)=\sin (|x+y|)$. Moreover, the random variable is Gaussian centered with variance $10^{-5}$ and covariance $10^{-6}$, so $w(x)$ can easily be simulated by Remark 6 .

In Tables 1 and 2, we see that the numbers of iterations are increasing rapidly with increasing $\tau$ for non-preconditioned systems for $\alpha=0.5$ and $\alpha=$ 1.5. This indicates that equation (6) is less well-conditioned as $\tau \rightarrow \infty$. Also, we see that the iterations performance of optimal circulant wavelet operators are smaller than super-optimal wavelet circulant operators. The reasons for these observations are stated in Remarks 2, 3 and the fast convergence for the PCG in section 3.4. Moreover, we give Table 3. regarding the relation between the number of iterations and the condition number of the matrices (when $\tau=25$, random vector $w_{n}$ is simulated by variance $10^{-5}$ and covariance $\left.10^{-6}\right)$.

Table 1. Iteration counts for example 1 with different preconditioners and $\alpha=0.5$

\begin{tabular}{|c||ccc|ccc|ccc|}
\hline & \multicolumn{3}{|c|}{$\tau=25$} & \multicolumn{3}{c|}{$\tau=75$} & \multicolumn{3}{c|}{$\tau=225$} \\
\hline $\mathrm{n}$ & Alg.W & Alg.S & Alg.O & Alg.W & Alg.S & Alg.O & Alg.W & Alg.S & Alg.O \\
\hline 40 & 47 & 12 & 12 & 43 & 11 & 10 & 63 & 13 & 12 \\
\hline 80 & 67 & 17 & 14 & 55 & 14 & 14 & 51 & 14 & 13 \\
\hline 120 & 95 & 21 & 17 & 86 & 23 & 19 & 63 & 19 & 19 \\
\hline 160 & 87 & 23 & 22 & 101 & 19 & 20 & 99 & 19 & 18 \\
\hline 200 & 93 & 25 & 23 & 116 & 21 & 20 & 107 & 25 & 19 \\
\hline
\end{tabular}


Table 2. Iteration counts for example 1 with different preconditioners and $\alpha=1.5$

\begin{tabular}{|c||ccc|ccc|ccc|}
\hline & \multicolumn{3}{|c|}{$\tau=25$} & \multicolumn{3}{|c|}{$\tau=75$} & \multicolumn{3}{c|}{$\tau=225$} \\
\hline n & Alg.W & Alg.S & Alg.O & Alg.W & Alg.S & Alg.O & Alg.W & Alg.S & Alg.O \\
\hline 40 & 33 & 12 & 10 & 61 & 12 & 13 & 47 & 14 & 12 \\
\hline 80 & 49 & 19 & 18 & 52 & 21 & 19 & 51 & 21 & 19 \\
\hline 120 & 53 & 24 & 21 & 49 & 25 & 21 & 67 & 23 & 21 \\
\hline 160 & 57 & 25 & 22 & 58 & 26 & 21 & 96 & 26 & 22 \\
\hline 200 & 69 & 31 & 27 & 71 & 32 & 29 & 68 & 29 & 24 \\
\hline
\end{tabular}

Table 3. Number of iterations and condition number for example 1 with different Algorithms

\begin{tabular}{|c|c|c|c|c|c|c|}
\hline$\alpha$ & \multicolumn{3}{|c|}{0.5} & \multicolumn{3}{|c|}{1.5} \\
\hline $\mathrm{n} \times \mathrm{n}$ & Alg. & Iteration & $\log _{2}$ ( Condition number) & Alg. & Iteration & $\log _{2}$ (Condition number) \\
\hline $10 \times 10$ & & 18 & $6.3 \times 10^{1}$ & & 16 & $2.3 \times 10^{1}$ \\
\hline $20 \times 20$ & W & 25 & $3.1 \times 10^{1}$ & W & 19 & $4.3 \times 10^{1}$ \\
\hline $30 \times 30$ & & 41 & $9.6 \times 10^{1}$ & & 32 & $1.9 \times 10^{2}$ \\
\hline $10 \times 10$ & & 4 & $4.6 \times 10^{-4}$ & & 6 & $2.1 \times 10^{-5}$ \\
\hline $20 \times 20$ & $\mathrm{~S}$ & 11 & $6.1 \times 10^{-4}$ & $\mathrm{~S}$ & 11 & $5.6 \times 10^{-4}$ \\
\hline $30 \times 30$ & & 12 & $9.0 \times 10^{-4}$ & & 14 & $5.1 \times 10^{-4}$ \\
\hline $10 \times 10$ & & 4 & $4.9 \times 10^{-5}$ & & 5 & $2.7 \times 10^{-6}$ \\
\hline $20 \times 20$ & $\mathrm{O}$ & 9 & $1.7 \times 10^{-5}$ & $\mathrm{O}$ & 7 & $6.4 \times 10^{-5}$ \\
\hline $30 \times 30$ & & 9 & $3.3 \times 10^{-4}$ & & 10 & $8.3 \times 10^{-5}$ \\
\hline
\end{tabular}

Example 2. We assume in $(20), K(x, y)=\frac{1}{4 \pi} a(x) \exp (-l(x, y))|x-y|^{-2}$, where $l(x, y)=|x+y|, \quad x, y \in G \subset \mathbb{R}^{3}$ be a part of sphere in $\mathbb{R}^{3}$ and its boundary $S$ is $\rho(\theta, \varphi)=(b \cos (\theta) \sin (\varphi), b \sin (\theta) \sin (\varphi), b \cos (\varphi))$ where $0 \leq$ $\theta \leq \frac{\pi}{2}, 0 \leq \varphi \leq \frac{\pi}{4}, 0<b \leq \infty$. From testing this example, Tables 4 , 5 are 6 give similar performance. When $a(x)=\exp (-|x|)$ and taking into consideration the similar assumptions for tables and figures of example (1). Numerical results for Example 2 confirm the conclusions drawn from Example 1.

Table 4. Iteration counts for example 2 with different preconditioners and $\alpha=0.5$

\begin{tabular}{|c||ccc|ccc|ccc|}
\hline & \multicolumn{3}{|c|}{$\tau=25$} & \multicolumn{3}{c|}{$\tau=75$} & \multicolumn{3}{c|}{$\tau=225$} \\
\hline $\mathrm{n}$ & Alg.W & Alg.S & Alg.O & Alg.W & Alg.S & Alg.O & Alg.W & Alg.S & Alg.O \\
\hline 40 & 56 & 23 & 18 & 52 & 22 & 20 & 63 & 21 & 20 \\
\hline 80 & 63 & 22 & 19 & 65 & 24 & 20 & 65 & 24 & 19 \\
\hline 120 & 70 & 25 & 22 & 74 & 25 & 21 & 87 & 26 & 21 \\
\hline 160 & 77 & 24 & 21 & 76 & 23 & 22 & 89 & 26 & 23 \\
\hline 200 & 112 & 32 & 25 & 96 & 34 & 27 & 95 & 31 & 24 \\
\hline
\end{tabular}


Table 5. Iteration counts for example 2 with different preconditioners and $\alpha=1.5$

\begin{tabular}{|c||ccc|ccc|ccc|}
\hline & \multicolumn{3}{|c|}{$\tau=25$} & \multicolumn{3}{|c|}{$\tau=75$} & \multicolumn{3}{c|}{$\tau=225$} \\
\hline $\mathrm{n}$ & Alg.W & Alg.S & Alg.O & Alg.W & Alg.S & Alg.O & Alg.W & Alg.S & Alg.O \\
\hline 40 & 44 & 18 & 17 & 54 & 21 & 18 & 56 & 23 & 18 \\
\hline 80 & 56 & 20 & 19 & 53 & 19 & 20 & 62 & 21 & 19 \\
\hline 120 & 75 & 22 & 23 & 77 & 24 & 23 & 83 & 23 & 21 \\
\hline 160 & 75 & 21 & 24 & 86 & 25 & 23 & 78 & 23 & 22 \\
\hline 200 & 74 & 23 & 25 & 67 & 22 & 26 & 84 & 28 & 23 \\
\hline
\end{tabular}

Table 6 . Number of iterations and condition number for example 2 with different Algorithms

\begin{tabular}{|c|c|c|c|c|c|c|}
\hline$\alpha$ & \multicolumn{3}{|c|}{0.5} & \multicolumn{3}{|c|}{1.5} \\
\hline $\mathrm{n} \times \mathrm{n}$ & Alg. & Iteration & $\log _{2}$ Condition number & Alg. & Iteration & $\log _{2}$ (Condition number) \\
\hline $10 \times 10$ & \multirow{3}{*}{ W } & 25 & $1.8 \times 10^{1}$ & \multirow{3}{*}{ W } & 19 & $6.1 \times 10^{1}$ \\
\hline $20 \times 20$ & & 72 & $2.3 \times 10^{1}$ & & 49 & $1.2 \times 10^{1}$ \\
\hline $30 \times 30$ & & 99 & $8.9 \times 10^{1}$ & & 80 & $0.2 \times 10^{2}$ \\
\hline $10 \times 10$ & \multirow{3}{*}{$\mathrm{S}$} & 7 & $3.3 \times 10^{-5}$ & \multirow{3}{*}{$\mathrm{S}$} & 8 & $1.7 \times 10^{-5}$ \\
\hline $20 \times 20$ & & 11 & $3.2 \times 10^{-4}$ & & 12 & $6.5 \times 10^{-5}$ \\
\hline $30 \times 30$ & & 12 & $9.6 \times 10^{-4}$ & & 11 & $7.8 \times 10^{-4}$ \\
\hline $10 \times 10$ & \multirow{3}{*}{$\mathrm{O}$} & 5 & $0.1 \times 10^{-6}$ & \multirow{3}{*}{$\mathrm{O}$} & 7 & $9.6 \times 10^{-7}$ \\
\hline $20 \times 20$ & & 7 & $3.5 \times 10^{-5}$ & & 11 & $2.6 \times 10^{-6}$ \\
\hline $30 \times 30$ & & 10 & $1.7 \times 10^{-5}$ & & 12 & $8.3 \times 10^{-5}$ \\
\hline
\end{tabular}

Numerical examples show that this approach is more effective than [20].

\section{REFERENCES}

1. A. Aldroubi and M. Unser, Families of multiresolutin and wavelet spaces with optimal properties, Numerical Functional Analysis and Optimization, 14, No. 5-6,(1993) pp. 417-446.

2. S.K. Biswas \& N.U. Ahmed, Stabilization of systems governed by the wave equation in the presence of distributed white noise, IEEE Transl. on Automatic control, AC. 30 (10) (1985).

3. S.H. Crandall \& W.Q. Zhu, Random Vibration, A survey of recent development journal of Applied Mechanics 50 (1980), pp.953-962.

4. G. David, Wavelets and Singular Integral on Curves and Surface, SpringerVerlag 1992.

5. A.A. Dorogavtsev, An extended stochastic integral for smooth functionals of white noise, Ukrainian Math. J. 41(1989), pp.1252-1258.

6. I. Gohberg \& I. Feldman, Convolution Equation and Projection Methods for their Solution, Transl. Math. Monographs, V, 41 (1974). 
7. I. Gohberg \& N. Krupnik, One-Dimensional Linear Singular Integral Equations, Volume I,II, Birkhäuser Verlag, Basel (1992).

8. I. Gohberg et al., Fast preconditioned conjugate gradient algorithms, SIAM J. Matrix Anal. Appl. 31 (1994), pp.429-443.

9. M.A. Golberg, Numerical Solution of Integral Equations, Plenum Press, New York (1990).

10. R.P. Kanwal,Linear Integral Equations Theory and Technique, Academic Press, New York (1971).

11. P.E. Kloeden \& E. Platen, Numerical Solution of Stochastic Differential Equations, Second corrected printing, Springer-Verlag Berlin (1995).

12. R. Kress, Linear Integral Equations, Springer-Verlag, Berlin (1989).

13. L.S. Langston, Heat transfer from multidimensional objects using onedimensional solutions for heat loss, Int. J. Heat Mass Transfer, 25 (1982), pp.149-150.

14. K. Maleknejad, Numerical experimentation with time-series methods for convolution integral equation, J. Computational and Applied Math. 31 (1990), pp.211-225.

15. K. Maleknejad \& D. Rostami V. F., Numerical solution of linear operator integral equation of the first kind by using methods of PCG, Lectures Note on Numerical Analysis, Plovdiv, Bulgaria, 4 (1995), pp.111-122.

16. M. Mitrea, Clifford Wavelets Singular Integrals and Hard Spaces, SpringerVerlag 1994.

17. G.E. Myers, Analytical Methods in Conduction Heat Transfer, McGrawHill, New York (1971).

18. P. M. Prenter, Splines and Variational Methods, John Wiley \& Sons, New York (1989).

19. J.J.Risler, Mathematical Methods for CAD, Cambridge University Press, Cambridge (1992).

20. D. Rostami V. F. \& K. Maleknejad, Preconditioners for solving stochastic boundary integral equation with weakly singular kernels, Computing 63 (1999), pp. 47-67.

21. D. Rostami V. F. \& K. Maleknejad, Preconditioned semilinear stochastic singular and hypersingular integral equations, Stochastic Analysis and applications, 18(6) (2000), pp. 949-956. 
22. T. Sekiguchi \& V. Shiota, $L_{2}$-theory of noncausal stochastic integrals, Math. Rep. Toyama Univ. 8 (1985), pp.119-195.

23. A.V. Skorokhod, On a generalization of the stochastic integral, Theory Random Processes 7 (1979), pp.118-127.

24. P.J. Schneider, Conduction Heat Transfer, Addison-Wesley, Reading, MA (1955).

25. E.E. Tyrtyshnikov \& V.V. Strela, Which circulant preconditioner is better?, Math of Comp. 65 (1996) pp.135-150.

26. M. Unser and T. Blu, Fractional splines and wavelets, SIAM Review, 42 NO. 1 (2000) pp. 43-67.

27. M. Unser and T. Blu, Construction of fractional spline wavelet bases, Proceedings of Wavelet Applications in Signal and Image, 3813, Processing VII, Denver Co, USA July 19-23, 1999,pp. 422-431.

28. R.J. Williams \& K.L. Chung, Introduction to Stochastic Integration, Birkhäuser, Boston (1990).

Received: September 2, 2006 